\title{
Still on the learning curve
}

\section{The Language of Shape: The Role of Curvature in Condensed Matter - Physics, Chemistry and Biology}

by Stephen Hyde, Sten Andersson, Kåre Larsson, Zoltan Blum, Tomas Landh, Sven Lidin and Barry W. Ninham

Elsevier: 1997. Pp. 383. Dfl370, $\$ 231.25$

\section{Alan L. Mackay}

A mixture of cyclohexane and aniline, when above room temperature, forms a single phase, but on cooling separates into two layers. On warming up, fluctuations in the interface increase in magnitude and wavelength, and the interface then vanishes at the critical temperature where the single phase becomes stable. Perhaps interfaces between scientific subjects are like that; The Language of Shape is an interface book, rather knobbly and uneven in its contents, but anticipating the coalescence of regions of physics, chemistry and biology. Although there is collective authorship by the seven writers, individual contributions are apparent, like large fluctuations in refractive index. They ask us to see surfaces rather than molecules.

The authors belong to groups in Canberra, Australia, and Lund, Sweden, which have been working to bring new mathematical and geometrical insights into chemistry with the aid of modern computer graphics and numerical calculations. Traditional chemistry has concentrated on the behaviour of isolated molecules in solution and has moved on to long chains and surface chemistry, and thence to solid-state behaviour. Following Linus Pauling, the three-dimensional structure of materials has usually been seen in terms of linked coordination polyhedra, but Stephen Hyde and his coauthors move up a level in the hierarchy of structure and emphasize the surfaces, interfaces and equipotentials produced by assemblies of molecules, where the salient variable is curvature. Curvature appears most clearly in dealing with sheets of molecules which can take up a great variety of topologies, many of them periodic in three dimensions.

Just as complex mathematical structures can arise from simple generating formulae - the Mandelbrot set is generated from $z^{\prime}=$ $z^{2}+c$ - so elaborate surfaces can appear from, for example, the requirement that the local mean curvature (the divergence of the unit vector normal to the surface) should be zero, plus boundary conditions. Complex long-range chemical structures may similarly arise from the requirements of simple local interactions.

Any such book must be compared with that published in 1917 by D'Arcy Thompson, On Growth and Form (Cambridge University Press, 1942, 2nd edn), which has long

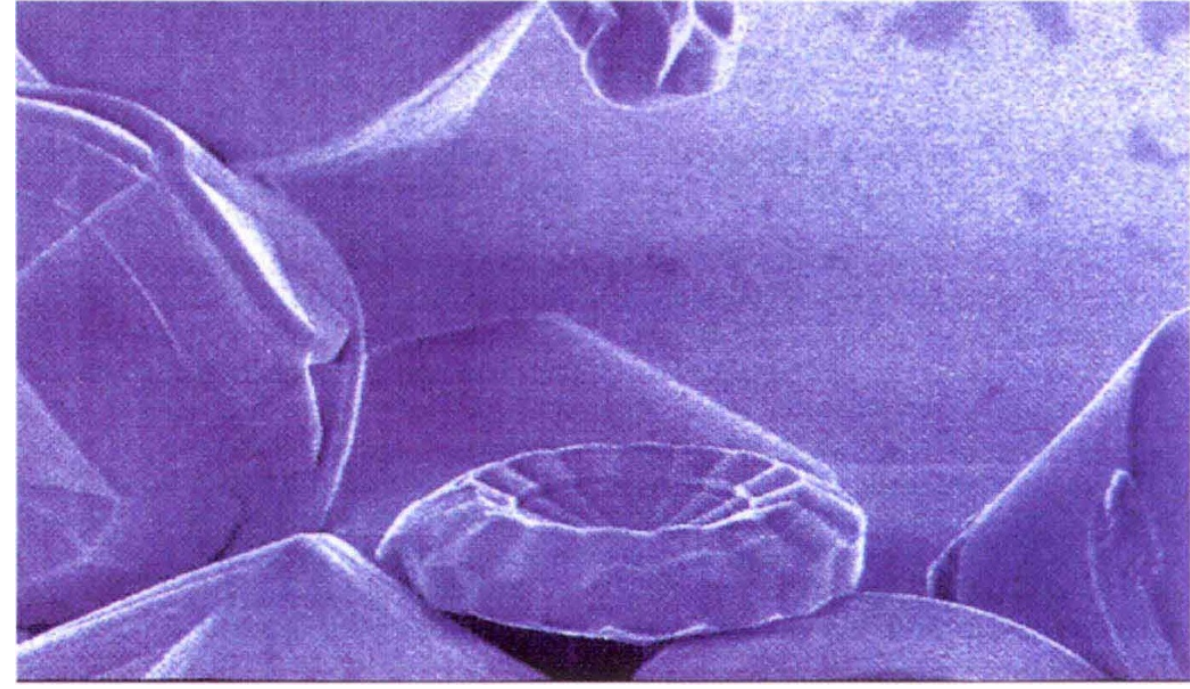

Complex forms due to spontaneous curvature developed during the precipitation of mesoporous silica.

demanded a sequel because Thompson hardly took note of atomicity. This new volume is not it, but it is a reconnaissance in strength of what may eventually appear. In 1936 Joseph Needham pointed out that "there are whole branches of mathematics dealing with the assessment of complexity, which no one has the ability or the imagination to make use of for grasping the biological situation" (Order and Life; MIT Press, 1968). In this latest book we have mainly the application of topology and the differential geometry of surfaces.

The book works through the basic mathematics of surfaces, then moves up from the atoms to the conceptual surfaces on which atoms can be seen to be concentrated, then to the forces of self-assembly and to the geometry of self-assembled systems to be found in liquid crystals, where individual molecules produce statistically anisotropic liquids. Copolymers may have very complex interfaces between the two phases. Lipids may form oriented monolayers and bilayers, leading to sheets and vesicles and ultimately to cell membranes. Crystals appear outmoded in comparison to the ingenuity of living systems, but periodicity sometimes reappears at higher levels of aggregation.

The longest chapter, based largely on the work of Tomas Landh, scours the literature for pictures of periodically structured cell membranes ('cubic membranes'). With computer reconstructions it convincingly explains hundreds of electron micrographs in terms of continuous sheets convoluted into the three-dimensionally periodic minimal surfaces, rather than as isolated units ordered in arrays. It would be hard to imagine the three-dimensional structure of a cabbage from a few two-dimensional sections, and this is also the case for the endoplasmic reticulum. We are everywhere encouraged to look anew at familiar structures and to see, for example, equipotential surfaces rather than discrete atoms (although at the level of single atoms Hyde's explanation of the martensitic transformation in steel remains an intriguing speculation).

Even more elaborate molecular assemblies are considered, and suggestions made for new attitudes to specific assemblies: protein molecules, starch grains, muscle and collagen. The authors are missionaries and seek to convert us to their ways of looking at familiar structures. Morphogenesis is today an extremely active topic, and intimations of a junction between synthetic chemistry and biology are appearing with the generation of frameworks resembling the shells of diatoms, where amorphous hydrated silica frameworks can be generated in the interstices of periodic arrays of large organic molecules. The organic material is then burnt out, leaving a three-dimensionally periodic lattice of silica with tunnels of width around $100 \AA$.

This work seeks to reinterpret the geometry of structures in new terms, asking us to see sheets rather than molecules. For this it is essential to re-educate one's spatial imagination with pictures, many of which are provided, but which represent intrinsically threedimensional structures for which one really needs to get off flat pages and make solid models. Moving up from the micro-world of atoms, this new outlook may yet meet, in the meso-world of the biological structures revealed by the electron microscope, that of D'Arcy Thompson moving down from the macro-world of the naturalist.

Alan L. Mackay is in the Department of Crystallography, Birkbeck College, University of London, Malet Street, London WC1E 7HX, UK. 